OPEN ACCESS

Edited by:

Maw Pin Tan,

University of Malaya, Malaysia

Reviewed by:

Lisa Robinson,

Newcastle upon Tyne Hospitals NHS

Foundation Trust, United Kingdom

Mario Ulises Pérez-Zepeda,

Instituto Nacional de Geriatría, Mexico

*Correspondence:

Kazuhiro Yasuda

kazuhiro-yasuda@aoni.waseda.jp

Specialty section:

This article was submitted to

Geriatric Medicine,

a section of the journal

Frontiers in Medicine

Received: 18 February 2018

Accepted: 01 May 2018

Published: 16 May 2018

Citation:

Yasuda K, Saichi K and Iwata H (2018)

Haptic-Based Perception-Empathy

Biofeedback Enhances Postural Motor

Learning During High-Cognitive Load

Task in Healthy Older Adults.

Front. Med. 5:149.

doi: 10.3389/fmed.2018.00149

\section{Haptic-Based Perception-Empathy Biofeedback Enhances Postural Motor Learning During High-Cognitive Load Task in Healthy Older Adults}

\author{
Kazuhiro Yasuda ${ }^{1 *}$, Kenta Saichi $^{2}$ and Hiroyasu Iwata ${ }^{2}$ \\ ${ }^{1}$ Research Institute for Science and Engineering, Waseda University, Tokyo, Japan, ${ }^{2}$ Graduate School of Creative Science \\ and Engineering, Waseda University, Tokyo, Japan
}

Falls and fall-induced injuries are major global public health problems, and sensory input impairment in older adults results in significant limitations in feedback-type postural control. A haptic-based biofeedback (BF) system can be used for augmenting somatosensory input in older adults, and the application of this BF system can increase the objectivity of the feedback and encourage comparison with that provided by a trainer. Nevertheless, an optimal BF system that focuses on interpersonal feedback for balance training in older adults has not been proposed. Thus, we proposed a haptic-based perception-empathy BF system that provides information regarding the older adult's center-of-foot pressure pattern to the trainee and trainer for refining the motor learning effect. The first objective of this study was to examine the effect of this balance training regimen in healthy older adults performing a postural learning task. Second, this study aimed to determine whether BF training required high cognitive load to clarify its practicability in real-life settings. Twenty older adults were assigned to two groups: BF and control groups. Participants in both groups tried balance training in the single-leg stance while performing a cognitive task (i.e., serial subtraction task). Retention was tested $24 \mathrm{~h}$ later. Testing comprised balance performance measures (i.e., $95 \%$ confidence ellipse area and mean velocity of sway) and dual-task performance (number of responses and correct answers). Measurements of postural control using a force plate revealed that the stability of the single-leg stance was significantly lower in the BF group than in the control group during the balance task. The BF group retained the improvement in the $95 \%$ confidence ellipse area $24 \mathrm{~h}$ after the retention test. Results of dual-task performance during the balance task were not different between the two groups. These results confirmed the potential benefit of the proposed balance training regimen in designing successful motor learning programs for preventing falls in older adults.

Keywords: older adults, postural control, sensory integration, haptic-based perception-empathy biofeedback, interpersonal feedback, motor learning 


\section{INTRODUCTION}

A previous study reported that $30-60 \%$ of healthy older adults experience falls annually and that $10-20 \%$ of such falls can result in injury, hospitalization, or death (1). Additionally, falls are known to cause loss of independence, a decline in the health status, and a decrease in quality of life (2). Moreover, fall-related injuries have been shown to be associated with high economic cost (3). Recurrent falls and impaired balance are among the most important risk factors for falls and should, thus, be addressed in fall prevention programs. Risk factors for postural instability in older adults vary and encompass various diseases, including abnormalities in balance and gait (4). Woollacott has suggested that borderline pathology in sensorimotor processes plays an important role in postural instability (5). Thus, the interplay between postural and voluntary movements on the basis of sensory control is significant in the performance of motor tasks (6). In this context, sensory input impairment in older adults must result in significant limitations in feedback-type motor control (7). For example, deficits in lower extremity joint proprioception or foot plantar sensory were found to be highly correlated with postural stability (8).

The technique of sensory substitution involves the use of a sensory modality to replace or augment another sensory modality (9). Previous studies have proposed various sensory substitution devices that provide auditory, vibrotactile, and multimodal biofeedback (BF) for countering age- and diseaserelated imbalance and decreasing fall risk (10-15). Vibrotactile feedback (VTF) was developed to provide individuals having balance problems with external information about their body movements in space. Generally, vibration cues are provided as feedback when body movements exceed a predefined threshold. The effects of VTF applied to the trunk for reducing postural sway in young, healthy subjects, and individuals with vestibular deficits have been validated in several previous studies (16-19). Moreover, studies have shown that VTF can reduce trunk tilt and improve gait performance in older adults $(15,20,21)$. Although a series of previous studies have substantiated the efficacy of VTF to a certain extent, recent studies have indicated that VTF can affect postural performance during dual tasks and can affect results of cognitive tasks $(20,22)$. This is probably because VTF requires participants to engage in higher cognitive processes to deal with the stimulus input. Specifically, older adults would be susceptible to decreased dual-task performance during postural control and gait tasks $(23,24)$.Thus, when attempting to apply VTF to actual daily life scenarios, this challenge should be seriously considered.

Studies involving skill science have reported that provision of summarized knowledge of results (KR) can help with motor learning $(25,26)$. Furthermore, previous studies have shown that learners desire to receive positive feedback after a "good" attempt at a task, and the findings indicate the function of feedback as a motivational tool (27-29). The use of BF might improve the quality of summarized KR or encouragement, as this approach allows objective observation of the motion characteristics of a trainee, according to $\mathrm{BF}$ information. However, no report has proposed an optimal BF system that would contribute to the refinement of interpersonal feedback between a trainer and trainee. The present study aimed to propose a haptic-based perception-empathy $\mathrm{BF}$ system capable of providing data on the center-of-foot pressure $(\mathrm{CoP})$ pattern during training to the trainee and trainer for enhancing the motor learning effect. First, the purpose of the present study was to investigate the impact of the proposed balance training regimen in healthy older adults performing postural learning task. Second, we aimed to reveal the effect of the proposed BF system on the cognitive load during balance training because this aspect is essential for clarifying its feasibility in real-life or clinical setting. We hypothesized that a balance training regimen using the proposed system would enhance balance motor learning in older adults.

\section{MATERIALS AND METHODS}

\section{Participants}

Recruitment of participants was conducted in the Shinjukuward, Tokyo area, and facilitated by the Shinjuku Silver Human Resources Center through advertising in local recruitment papers. At the initial visit, a screening physical examination and hearing test were performed. Inclusion criteria were age $\geq 65$, having sufficient communication abilities to understand the instructions provided by the experimenter, living independently in the community, being able to maintain balance in a single-leg stance for $>30 \mathrm{~s}$, being able to walk without an aid, being free of neurological or musculoskeletal issues that might influence postural control or cognition (cerebrovascular accident, brain trauma, Parkinson's disease, acute illness, significant orthopedic disability, etc.), having a mini-mental state examination score $>20$ (no dementia), and having the ability to sense vibrations of the BF system. Moreover, participants were excluded if they had severe visual impairment or hearing deficit, nerve damage, body pain, a history of fainting, or a body mass index $>30 \mathrm{~kg} / \mathrm{m}^{2}$.

Twenty older adults participated in this study, and the participants were randomly allocated to the BF or control groups by a third-party institution (Silver Human Resources Center). An initial sample size of 8 per group was suggested for the pilot study with G-power (30). This was later amended to 10 per group so that the study could be completed with human resources.

\section{Ethical Statements}

All procedures were approved by Waseda University Ethics Committee for Human Research. Prior to participation, each participant signed an informed consent form approved by an institutional review board (approval number: 2012-247).

\section{BF System Overview}

The BF system includes a Nintendo Wii balance board (WBB) (Nintendo Co., Ltd., Kyoto, Japan), a wearable vibrotactile BF device, and a personal computer having a custom-programmed software (Visual Studio; Microsoft Corp., Redmond, WA, USA). The software allows the BF threshold to be recorded and manipulated. We selected the WBB to ensure high usability in a clinical environment, as previous studies have shown that the WBB has good-to-excellent reliability with regard to functional balance performance in healthy older adults $(31,32)$. The wearable BF device assesses the perception of the pattern 
of CoP displacement at the pelvis (i.e., anterior and posterior superior iliac spine) during the postural task (Figure 1A). To alleviate cognitive loads on older adults and detect the direction of CoP motion, only four tactile vibrators were used around the participant's pelvis (Figure 1A). Each vibrator (frequency: $80 \mathrm{~Hz}$ ) was activated when $\mathrm{CoP}$ exceeded a predefined circular threshold (33). We used the following approach to determine the predefined circular threshold (33). First, the 95\% confidence circle area (34) was measured during a 30-s stance. Second, the target area was defined by $90 \%$ of the premeasured $95 \%$ confidence circle area. Using this value, the activated vibrators made the participants aware of the direction of their body sway. In older adults, deficits in lower extremity joint proprioception or foot plantar sensory were found to be highly correlated with postural stability (8). In the present BF system, body sway data is augmented with vibrators that are attached to the pelvic girdle.

This system provides $\mathrm{BF}$ on the CoP pattern during training to not only the trainee but also the trainer (physical therapist or coach) (Figure 1B). Thus, the postural sway pattern information is shared. Studies have indicated that appropriate instructions to modify movement patterns (3537 ) and appropriate motivation for motor learning should be provided to participants $(27,28)$. However, it is not possible to objectively understand the features of correct movement patterns in real time according to only a trainer's observation. In particular, it is not possible to detect subtle postural movements. Thus, instructions tend to depend on a trainer's experience. As this system shares BF information, it (a) enables the trainer to precisely monitor the trainee's CoP patterns and provide accurate summary feedback and (b) makes it possible to effectively provide encouraging feedback by sharing the information that the balance performance has achieved the objective. Thus, all trainers can accurately and immediately instruct and encourage participants during balance training based on objective $\mathrm{BF}$ information, not on subjective evaluation.

\section{Protocol and Postural Task}

In this study, we selected single-leg stance tasks to observe their effects on postural motor learning because single-leg stance tasks are more challenging tasks that are predictive of balance problems and better indicators of fall risk in older adults (38-40).

Measurement of postural sway comprised three phases: baseline test, postural training (day 1), and retention test (day 2) (Figure 2). Before the baseline test evaluation, participants were given 10 practice sessions ( $30 \mathrm{~s}$ each) for familiarization with the single-leg stance task. After this practice session, five baseline measurements ( $30 \mathrm{~s}$ each) of postural sway in the single-leg stance were obtained as a baseline test. The predefined circular threshold was determined at the last baseline measurement.

fixed eye-level target test, participants proceed to the postural training session. Each participant stood barefoot on the WBB with eyes open while looking at a fixed eye-level target placed $\sim 2 \mathrm{~m}$ away. A training session is composed of 15 performances ( $30 \mathrm{~s}$ each), with a rest interval of 1 min between each performance. For both groups, the participants were asked to minimize postural sway to avoid activating the vibrators.
To synchronize the lengths of time required for providing feedback to the BF and control groups, feedback was only provided once every three trials during 1-min intervals (5 min in total). The BF group received specific feedback from the trainer, and encouragement was provided during training sessions. Shared haptic feedback information was only used to provide feedback and encouragement. Specifically, feedback regarding the body sway pattern was provided (e.g., "The body is leaning toward the forward right during training," "It takes time to return to the left side from the right side," or "The body is swaying forward and backward on the right side"). If appropriate balance was achieved, encouragement was provided with words such as "good" and "that's better." The control group also underwent postural control tasks under the same experimental conditions, except the use of BF. The control group received feedback about postural sway pattern every three trials. This feedback and encouragement were based on the observation of the trainer. Feedback to the participants was provided by the same licensed physical therapist.

On day 2 ( $\sim 24 \mathrm{~h}$ between visits), the participants underwent the same initial approach (10 postural tasks). Then, a retention test was performed. It involved five tasks ( $30 \mathrm{~s}$ each), with a rest interval of $1 \mathrm{~min}$ between tasks.

\section{Secondary Cognitive Task: A Digit Subtraction Task}

To evaluate the effect of dual tasks during the postural tasks, a digit subtraction task was used during the single-leg stance task $(41,42)$. The participants were told to count aloud backward by seven from a number that was determined through the selection of a card with a random number from 125 to 250. For each postural trial, the participants were told to randomly select a different card from a pack of cards. The participants were shown the selected card for $5 \mathrm{~s}$ before the start of the balance task. There were no practice or familiarization trials for the additional task in order to prevent the participants from learning the secondary task.

\section{Outcome Measurements Postural Performance}

Two representative dependent variables were used for describing the participant's postural stability (43). First, 95\% confidence ellipse area was used as a measure of CoP spatial variability (43). It represents the $95 \%$ bivariate confidence ellipse area that is expected to cover about $95 \%$ of points on the CoP path. Second, the mean velocity of CoP displacement $(\mathrm{mm} / \mathrm{s})$ was considered. It represents the total distance covered by $\mathrm{CoP}$ (total sway path) divided by the test duration $(43,44)$.

\section{Secondary Cognitive Task}

Cognitive performance is considered as the number of correct arithmetic calculations (45). When participants simultaneously performed the cognitive and postural tasks (dual-task trials), dual-task scores were calculated. When participants performed the cognitive task, single-task scores were calculated. During the single task, each participant was told to subtract seven from a random number as many times as possible in $30 \mathrm{~s}$. 


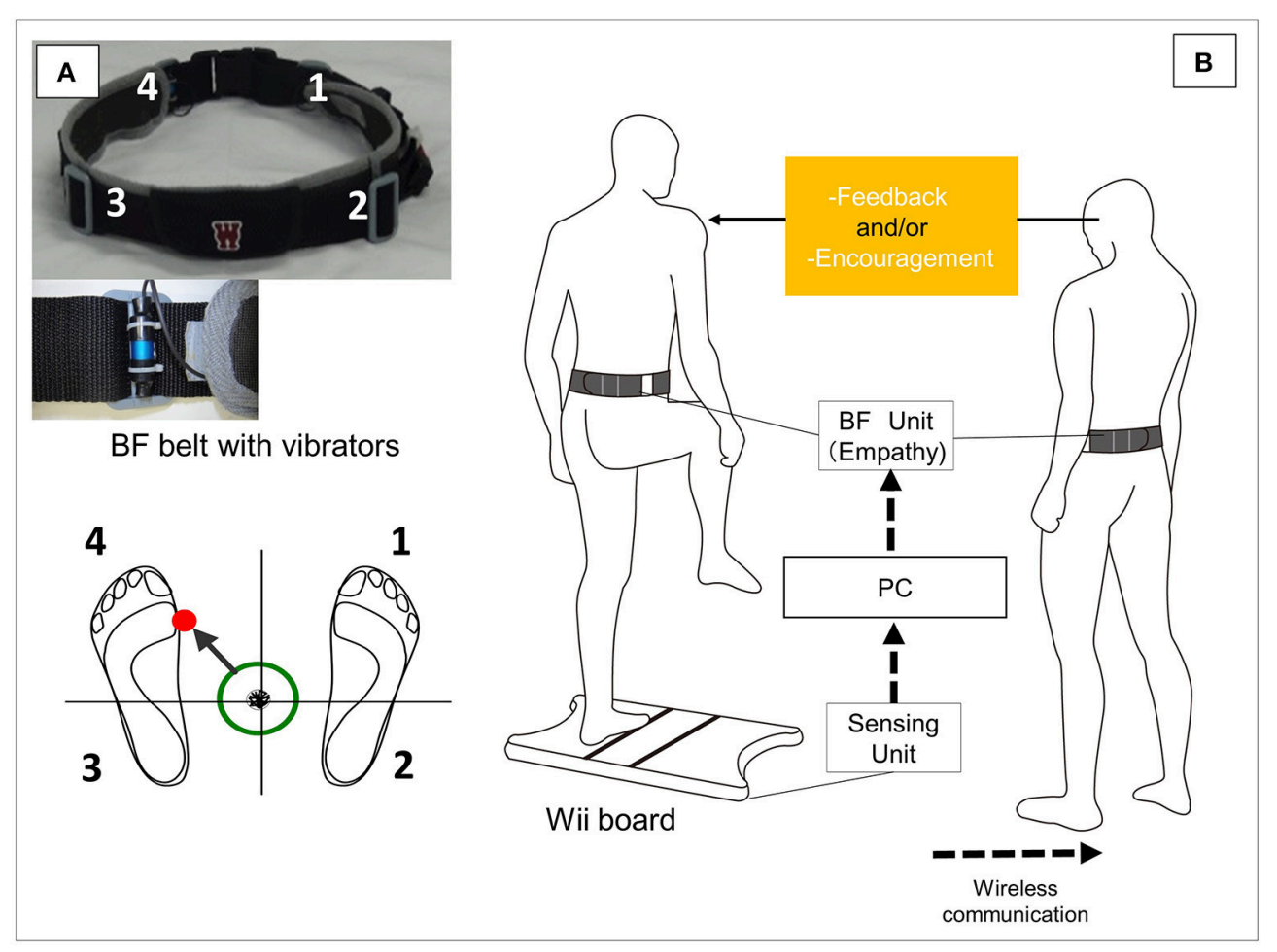

FIGURE 1 | Overview of the biofeedback system. (A) When the center-of-foot pressure (CoP) exceeds the predefined threshold area, vibrators on the participant's pelvic belt are activated in the corresponding CoP direction (as indicated in the illustration, when CoP shifts to the front left, the vibrator on the front left is activated). (B) During balance training, the vibrators on the trainee's and trainer's pelvic belts are simultaneously activated corresponding to the trainee's CoP direction. Based on the shared information, the trainer is able to provide appropriate feedback and encouragement.

Verbalizations were recorded from a digital video camera with a built-in microphone and then analyzed in Adobe Premiere Pro CC (Adobe Systems, San Jose, CA).

\section{Analysis}

\section{Postural Performance}

Prior to the analysis, the motor performance score was separately normalized (\%) to the baseline for each subject (46). Then, the 95\% confidence ellipse area and MV values were normalized to the baseline test for each group. As a result of this normalization, all baseline values for the $95 \%$ confidence ellipse area and MV were $\fallingdotseq 1$.

To assess an improvement associated with adaptation on day 1 of training, the postural training session involved 15 trials of the single-leg stance task. Similarly, retention (day 2) was assessed in five trials of the single-leg stance task. Normality was assessed using the Shapiro-Wilk test, and if violation of normality was noted, a non-parametric test was considered. Scores were analyzed in a univariate analysis of variance (ANOVA) (47). For the day 1 training session, scores were analyzed in 2 groups $\times 15$ trial ANOVA with repeated measures on the last factor (47). The retention of improvement was assessed by comparing the results of trials $1-5$ on day 1 and the results of the same trials on day 2 , using a paired samples $t$-test or the Wilcoxon signed-rank test $(48,49)$. Alpha was set at $p=0.05$.

\section{Cognitive Performance}

Prior to the analysis, the number of correct digits verbalized during dual-task trials was normalized to that verbalized during a proportional time period in a single task (i.e., subtraction task in the seating position) (45). A two-tailed, independent $t$-test or Mann-Whitney test was used for determining if cognitive performance in the $\mathrm{BF}$ and control groups differed during 15 trials.

\section{RESULTS}

\section{Participant Characteristics}

Participants' characteristics are described in Table 1. There were no differences between the $\mathrm{BF}$ and control group regarding basic characteristics of the participants.

\section{Postural Performance}

Results of the two-way ANOVA for the postural sway parameters are depicted in Table 2.

\section{5\% Confidence Ellipse Area}

Baseline scores between $\mathrm{BF}$ and control groups were not different (Figure 3, left). For the postural performance on day 1, the main effect in the groups was significant $\left[F_{(1,18)}=53.30, p<0.0001\right]$. Throughout the training, the $\mathrm{BF}$ group outperformed the control group (Figure 3, middle). The main effect of the trials 


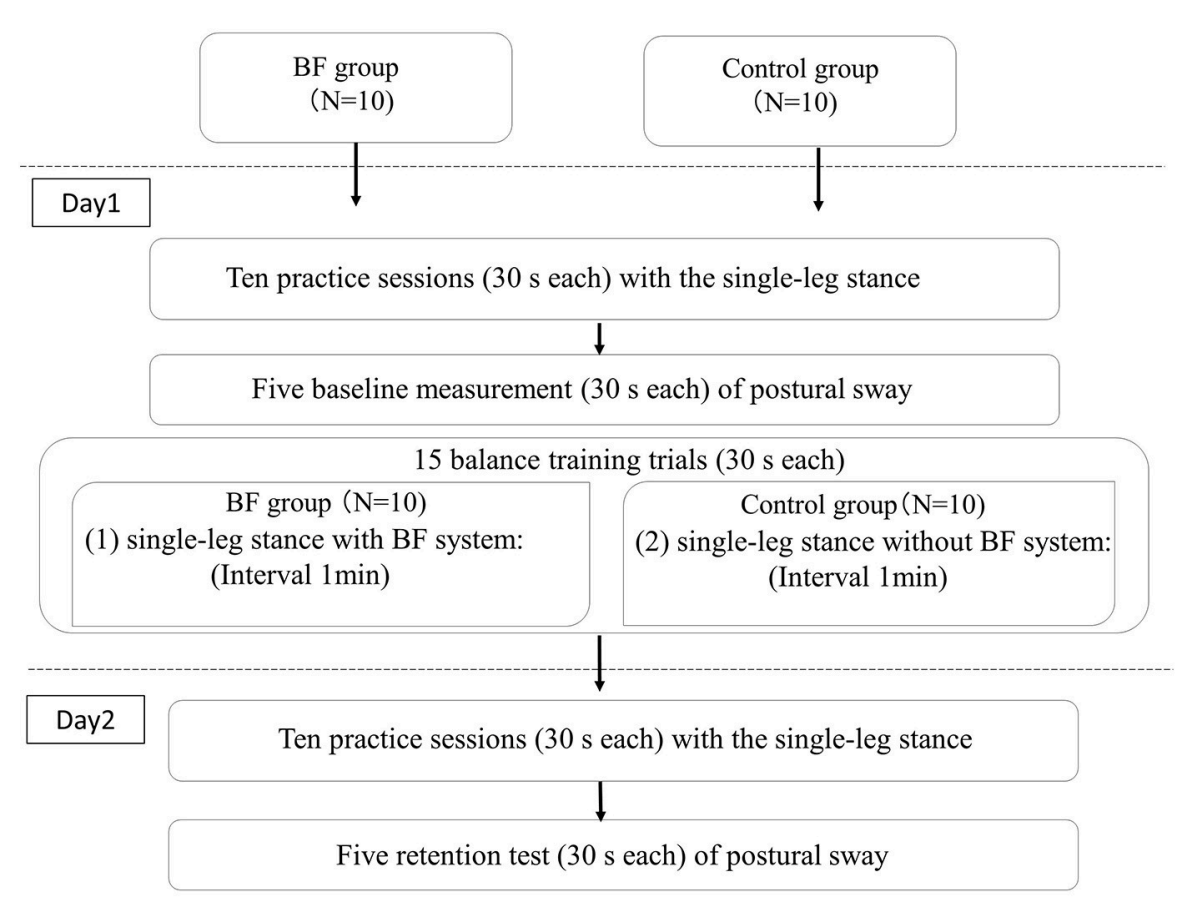

FIGURE 2 | Flow chart of the experimental procedure.

TABLE 1 | Participant characteristics.

\begin{tabular}{llll}
\hline & BF group $(\boldsymbol{n}=\mathbf{1 0})$ & Control group $(\boldsymbol{n}=\mathbf{1 0})$ & $\boldsymbol{p}$-value \\
\hline Sex (n, females) & 5 & 5 & \\
Age (y) & $71.2 \pm 2.4$ & $72.7 \pm 3.4$ & 0.9370 \\
Weight (kg) & $59.0 \pm 13.7$ & $55.6 \pm 5.0$ & 0.4717 \\
Height (cm) & $161.9 \pm 6.6$ & $158.5 \pm 6.2$ & 0.2472 \\
Leg length (cm) & $81.2 \pm 4.5$ & $79.3 \pm 4.4$ & 0.3729
\end{tabular}

Values are denoted in mean $\pm S D$. BF, biofeedback.

$\left[F_{(14,252)}=2.425, p=0.0033\right]$ and interactions between the groups and trials were significant $\left[F_{(14,252)}=3.333, p<0.0001\right]$. Follow-up analyses indicated that the BF group decreased their sway area from trial 1 to trial $9(p=0.000138$.), from trial 1 to trial $12(p<0.0001)$, from trial 1 to trial $13(p<0.0001)$, from trial 1 to trial 14 ( $p=0.000114)$, and from trial 1 to trial 15 ( $p<0.0001)$, whereas the control group showed no change across trials. Adaptations were retained on day 2 for the BF group $(t=4.41, p=0.0017)$ but not for the control group $(t=1.48$, $p=0.1732$ ).

\section{Mean Velocity of Sway}

Baseline scores were not different between the BF and control groups (Figure 4, left). For postural performance on day 1 , the main effect in the groups was significant $\left[F_{(1,18)}=8.802, p=0.0083\right]$. Throughout the training, the $\mathrm{BF}$ group outperformed the control group (Figure 4, middle). The main effect of trials $\left[F_{(14,252)}=9.325, p<0.0001\right]$ and
TABLE 2 | Two-way ANOVA results of the 95\% confidence ellipse area and mean velocity of sway in the experiment.

\section{POSTURAL SWAY PARAMETERS}

\begin{tabular}{|c|c|c|c|c|}
\hline \multicolumn{5}{|c|}{$95 \%$ CONFIDENCE ELLIPSE AREA $(n=10)$} \\
\hline Source of variation & DF & MS & $\boldsymbol{F}$ & $\boldsymbol{P}$ \\
\hline Group (A) & 1 & 20.63 & 53.30 & $<0.0001^{* *}$ \\
\hline Trials (B) & 14 & 0.1374 & 2.425 & $0.0033^{\star \star}$ \\
\hline$A \times B$ & 14 & 0.1888 & 3.33 & $<0.0001^{*}$ \\
\hline Residual & 252 & 0.05665 & & \\
\hline Total & 281 & & & \\
\hline \multicolumn{5}{|c|}{ MEAN VELOCITY OF SWAY $(n=10)$} \\
\hline Source of variation & DF & MS & $\boldsymbol{F}$ & $\boldsymbol{P}$ \\
\hline Group (A) & 1 & 1.497 & 8.80 & $0.0083^{\star \star}$ \\
\hline Trials (B) & 14 & 0.07325 & 9.33 & $<0.0001^{\star \star}$ \\
\hline$A \times B$ & 14 & 0.02353 & 2.99 & $0.0003^{\star \star}$ \\
\hline Residual & 252 & 0.007855 & & \\
\hline
\end{tabular}

Total

281

$p$-value derived from ANOVA for the main effects of group and trials and interaction between group and trials. DF, degrees of freedom; MS, mean square. ${ }^{* *} p<0.01$.

the interaction between the groups and trials were significant $\left[F_{(14,252)}=2.996, p=0.0003\right]$. Follow-up analyses indicated that the BF group had decreased sway velocity from trial 1 to trial 8 $(p<0.0001)$, from trial 1 to trial $9(p<0.0001)$, from trial 1 to trial $10(p<0.0001)$, from trial 1 to trial $11(p<0.0001)$, from trial 1 to trial $12(p<0.0001)$, from trial 1 to trial $13(p<0.0001)$, from trial 1 to trial $14(p<0.0001)$, and from trial 1 to trial 


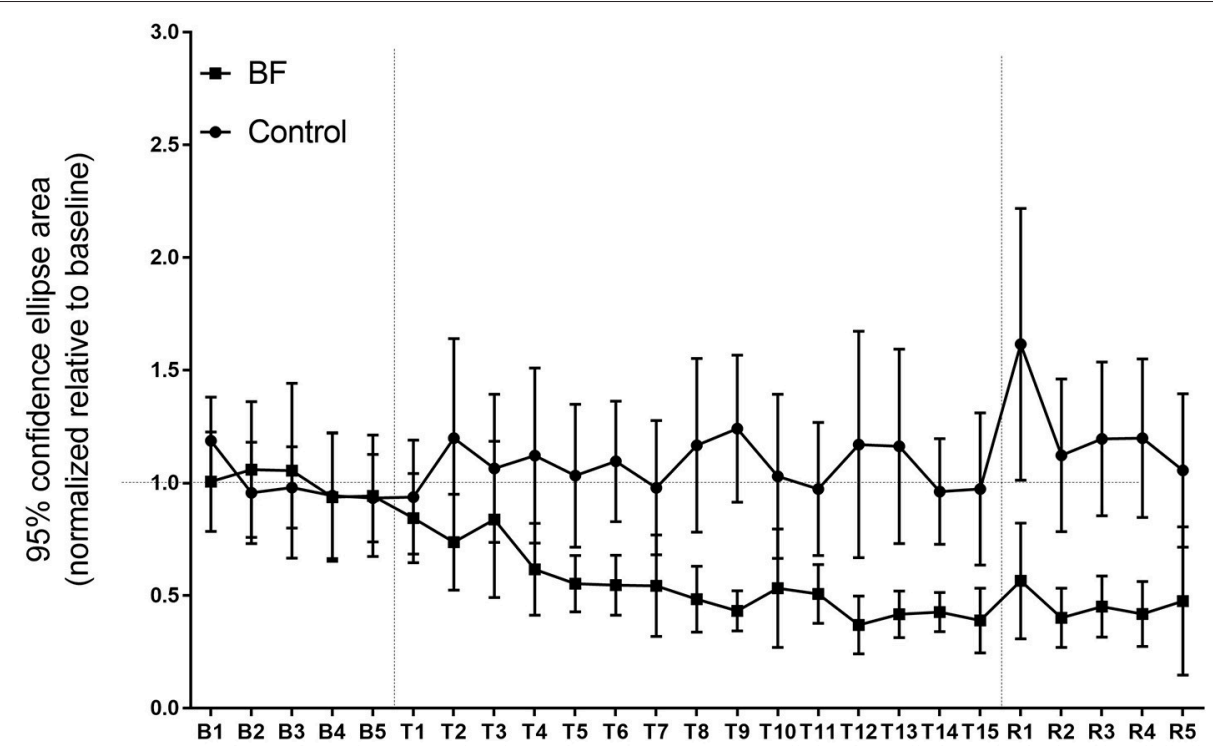

FIGURE 3 | Ninety-five percent confidence ellipse area in the experiment (higher scores reflect greater spatial variability). Error bars indicate standard deviation.

$15(p<0.0001)$, whereas the control group had decreased sway velocity from trial 2 to trial $14(p<0.0001)$, from trial 3 to trial $14(p=0.000162)$, and from trial 4 to trial $14(p<0.0001)$. The adaptations were not retained on day 2 for the BF group $(t=1.36$, $p=0.20)$ and control group $(t=1.06, p=0.3426)$.

\section{Cognitive Performance}

Regarding cognitive performance, no significant difference was found between the $\mathrm{BF}$ and control groups in the number of answer $(t=0.60, p=0.5567)$ and percentage of correct answer $(t=1.32, p=0.2004$; Figure 5).

\section{DISCUSSION}

This initial study has shown that using the proposed system, the $\mathrm{BF}$ group was more effective in adapting to the postural control tasks than the control group, and the older participants retained these improvements over $24 \mathrm{~h}$ only for spatial variability, but not for the mean velocity of sway. Moreover, cognitive loads applied to the participants were not significantly different between the $\mathrm{BF}$ and control groups, suggesting that the cognitive burden of using the BF system is low. These results increase expectations that the BF system proposed in this study will contribute to developing efficient motor learning modalities incorporating postural control tasks for older people. Indeed, this system is anticipated to be highly practical given its low cognitive burden.

Several factors might have been associated with the beneficial effect with regard to the balance performance observed in this study. The addition of $\mathrm{BF}$ might have resulted in an increase in the accuracy of motion correction during BF training (33). Additionally, empathy feedback might have had several effects, including (a) realization of accurate summary feedback from the trainer and (b) strengthening of the reward with encouragement provided by the trainer. With regard to the realization of accurate summary feedback from the trainer, it has been shown that motor learning is facilitated by providing summarized $\mathrm{KR}(25,26)$. The quality of summarized KR might have been improved by empathy $\mathrm{BF}$, as the trainer could objectively determine the motion characteristics of older adults considering the vibration information with empathy BF. With regard to strengthening of the reward with encouragement provided by the trainer, studies involving skill science have shown that learners desire to receive feedback after a good rather than poor attempt, and the findings indicate the function of feedback as a motivational tool $(27,28)$. Furthermore, rewards have been shown to enhance memory retention across multiple motor learning models in healthy subjects (29). Considering that encouragement can be provided according to the shared $\mathrm{BF}$ of an objective task and that encouragement can be provided when the defined exercise target is achieved, shared BF might have enhanced the effect of encouragement as a reward.

In this study, participants solve some mental arithmetic tasks of subtraction during the postural control tasks, and performance of the BF group was compared with that of the control group. Consequently, the use of $\mathrm{BF}$ in the $\mathrm{BF}$ group did not negatively affect the performance of dual tasks compared with that of the control group. Previous studies found that secondary cognitive tasks (reaction time) significantly increased while using VTF in younger and older adults $(20,22)$. Older adults had a larger increase in reaction time than younger adults, suggesting that greater attentional demands were required in older adults when using VTF information. Given these results, the authors have pointed out that future training protocols for VTF should consider the 


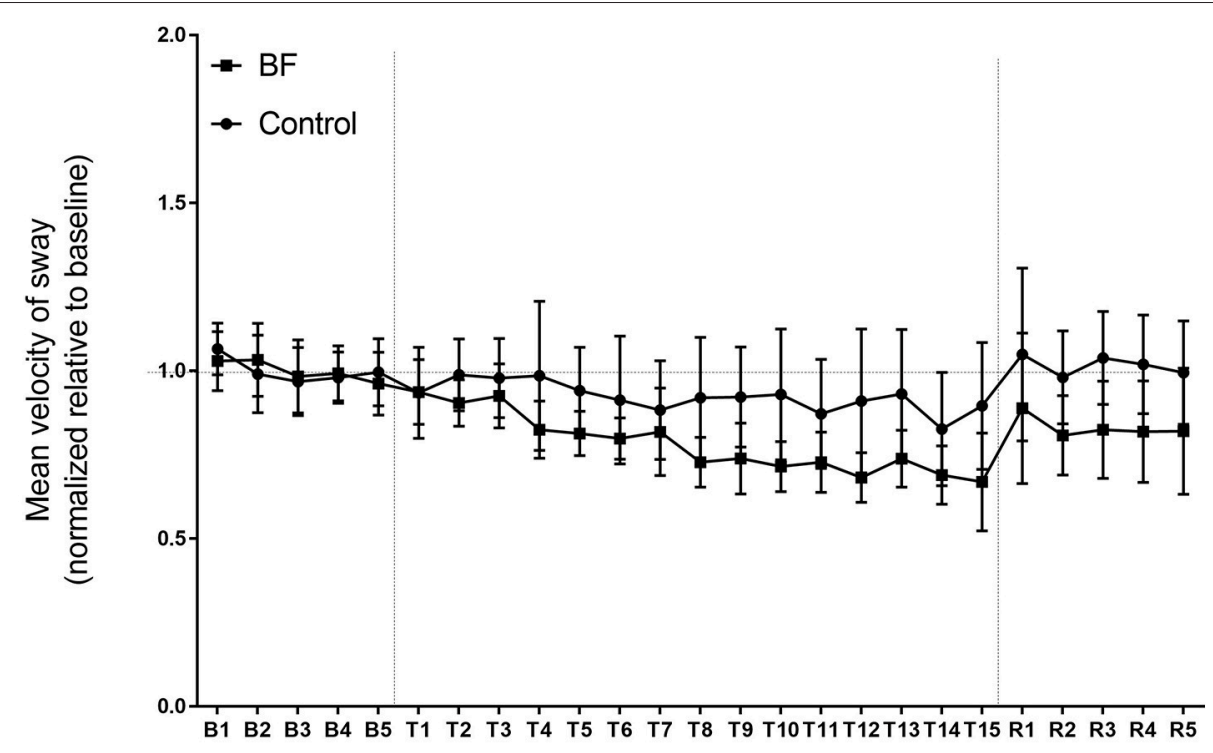

FIGURE 4 | The mean velocity of sway in the experiment (higher scores reflect greater quantity of postural sway). Error bars indicate standard deviation.
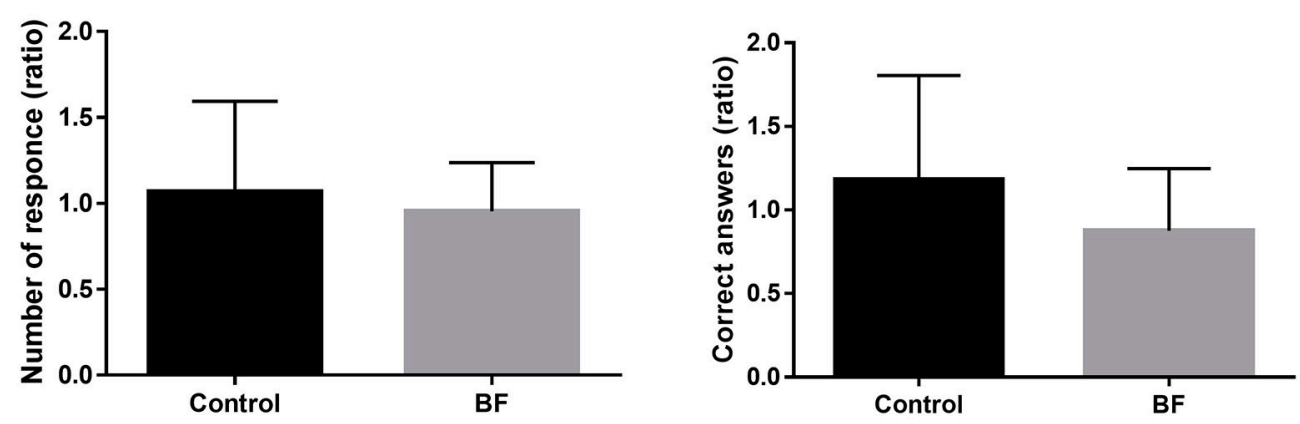

FIGURE 5 | Dual-task costs (as a ratio in performance compared with single-task performance) (mean \pm standard deviation).

effect of aging (20). Our system uses a minimum number of vibration cues (four points) and allows the instructor to utilize and provide BF information identical to the summarized KR. These attributes might have helped mitigate cognitive burden during training. Results of this study apparently suggest that the proposed BF training system can provide training regimens with relatively low cognitive burdens (or negative effects associated with postural control tasks) even for older participants who are susceptible to adverse effects of dual tasks. However, further validation using different sets of tasks is necessary because repercussions of cognitive loads are contingent on the characteristics of each cognitive task $(23,24)$.

Several limitations have been noted. First, it is impossible to strictly quantify the extent of vibration feedback, and the guidance/feedback derived from BF empathy affected the results of the present study. To clarify this point, basic comparative research on single BF vs. dual BF will be required. Second, the sample size was small, and further rigorous studies with a large sample size are required to confirm these results. Finally, although this study evaluated the feasibility of the device, practice was performed for only 1 day and retention was evaluated for only $24 \mathrm{~h}$. Future studies with a more advanced design are required to assess long-term balance training programs and to investigate whether improvements during training can be retained over a long period.

In conclusion, the present study demonstrated that the BF group more efficiently adapted to the balancing tasks than the control group that did not use BF, and older participants retained the improvement of postural spatial variability over $24 \mathrm{~h}$ only for spatial variability but not for the mean velocity of sway. With regard to the cognitive costs during the learning tasks, no significant difference was observed between the BF and control groups, thus suggesting the low cognitive cost of our system. The results indicated that the initial feasibility of the proposed balance training method in enhancing the motor learning effect highlights the potential use of the proposed BF systems in the field or clinical setting. 


\section{AUTHOR CONTRIBUTIONS}

KY designed this study, acquired and analyzed data, and drafted the manuscript. KS made substantial contributions to the acquisition and analysis of the data. HI was involved in the conception of the system and design of the study. The authors have read and concurred with the content of the final manuscript. No one who qualifies for authorship has been omitted from the list.

\section{FUNDING}

This study was supported by the Japan Society for the Promotion of Science (JSPS), Grant-in-Aid for Scientific

\section{REFERENCES}

1. Rubenstein LZ. Falls in older people: epidemiology, risk factors and strategies for prevention. Age Ageing (2006) 35:ii37-41. doi: 10.1093/ageing/afl084

2. Roe B, Howell F, Riniotis K, Beech R, Crome P, Ong BN. Older people and falls: health status, quality of life, lifestyle, care networks, prevention and views on service use following a recent fall. J Clin Nurs. (2009) 18:2261-72. doi: 10.1111/j.1365-2702.2008.02747.x

3. Arai H, Ouchi Y, Toba K, Endo T, Shimokado K, Tsubota K, et al. Japan as the front-runner of super-aged societies: perspectives from medicine and medical care in Japan. Geriatr Gerontol Int. (2015) 15:673-87. doi: 10.1111/ggi.12450

4. Blaszczyk JW, Hansen PD, Lowe DL. Evaluation of the postural stability in man: movement and posture interaction. Acta Neurobiol Exp. (1993) 53:155-60.

5. Woollacott MH, Shumway-Cook A. Changes in posture control across the life span-a systems approach. Phys Ther. (1990) 70:799-807. doi: $10.1093 / \mathrm{ptj} / 70.12 .799$

6. Massion J. Movement, posture and equilibrium: interaction and coordination. Prog Neurobiol. (1992) 38:35-56.

7. Welford AT. Between bodily changes and performance: some possible reasons for slowing with age. Exp Aging Res. (1984) 10:73-88. doi: 10.1080/03610738408258548

8. Lord SR, Clark RD, Webster IW. Postural stability and associated physiological factors in a population of aged persons. J Gerontol. (1991) 46:M69-76. doi: 10.1093/geronj/46.3.M69

9. Bach-y-Rita P, Collins CC, Saunders FA, White B, Scadden L. Vision substitution by tactile image projection. Nature (1969) 221:963-4. doi: $10.1038 / 221963 \mathrm{a} 0$

10. Dozza M, Horak FB, Chiari L. Auditory biofeedback substitutes for loss of sensory information in maintaining stance. Exp Brain Res. (2007) 178:37-48. doi: 10.1007/s00221-006-0709-y

11. Honegger F, Hillebrandt IM, van den Elzen NG, Tang KS, Allum JH. The effect of prosthetic feedback on the strategies and synergies used by vestibular loss subjects to control stance. J Neuroeng Rehabil. (2013) 10:115. doi: 10.1186/1743-0003-10-115

12. Nanhoe-Mahabier W, Allum JH, Pasman EP, Overeem S, Bloem BR. The effects of vibrotactile biofeedback training on trunk sway in Parkinson's disease patients. Parkinson Relat Disord. (2012) 18:1017-21. doi: 10.1016/j.parkreldis.2012.05.018

13. Horak FB, Dozza M, Peterka R, Chiari L, Wall C III. Vibrotactile biofeedback improves tandem gait in patients with unilateral vestibular loss. Ann NY Acad Sci. (2009) 1164:279-81. doi: 10.1111/j.1749-6632.2008.03707.x

14. Zijlstra A, Mancini M, Chiari L, Zijlstra W. Biofeedback for training balance and mobility tasks in older populations: a systematic review. J Neuroeng Rehabil. (2010) 7:58. doi: 10.1186/1743-0003-7-58

15. Davis JR, Carpenter MG, Tschanz R, Meyes S, Debrunner D, Burger J, et al. Trunk sway reductions in young and older adults
Research (C) No. 17K01875 and Grant-in-Aid for Junior Researchers, Research Institute for Science and Engineering, Waseda University [Technological Research Section: 17C13] and the Global Robot Academia Institute, Waseda University [FY2017].

\section{ACKNOWLEDGMENTS}

The authors thank the Shinjuku Silver Human Resources Center for assisting in participant recruitment and screening. The authors would also like to thank Zenyu Ogawa for his support in designing the hardware. The experiments were conducted in the Global Robot Academia Laboratory at the Waseda University.

using multi-modal biofeedback. Gait Posture (2010) 31:465-72. doi: 10.1016/j.gaitpost.2010.02.002

16. Basta D, Ernst A. [Vibrotactile neurofeedback training with the Vertiguard(R)-RT-system. A placebo-controlled double-blinded pilot study on vestibular rehabilitation]. HNO (2011) 59:1005-11. doi: 10.1007/s00106-011-2346-4

17. Bechly KE, Carender WJ, Myles JD, Sienko KH. Determining the preferred modality for real-time biofeedback during balance training. Gait Posture (2013) 37:391-6. doi: 10.1016/j.gaitpost.2012.08.007

18. Dozza M, Wall C III, Peterka RJ, Chiari L, Horak FB. Effects of practicing tandem gait with and without vibrotactile biofeedback in subjects with unilateral vestibular loss. J Vestib Res. (2007) 17:195-204.

19. Wall C III, Kentala E. Effect of displacement, velocity, and combined vibrotactile tilt feedback on postural control of vestibulopathic subjects. $J$ Vestib Res. (2010) 20:61-9. doi: 10.3233/VES-2010-0369

20. Haggerty S, Jiang LT, Galecki A, Sienko KH. Effects of biofeedback on secondary-task response time and postural stability in older adults. Gait Posture (2012) 35:523-8. doi: 10.1016/j.gaitpost.2011.10.359

21. Wall C III, Wrisley DM, Statler KD. Vibrotactile tilt feedback improves dynamic gait index: a fall risk indicator in older adults. Gait Posture (2009) 30:16-21. doi: 10.1016/j.gaitpost.2009.02.019

22. Verhoeff LL, Horlings CG, Janssen LJ, Bridenbaugh SA, Allum JH. Effects of biofeedback on trunk sway during dual tasking in the healthy young and elderly. Gait Posture (2009) 30:76-81. doi: 10.1016/j.gaitpost.2009.03.002

23. Fraizer EV, Mitra S. Methodological and interpretive issues in posturecognition dual-tasking in upright stance. Gait Posture (2008) 27:271-9. doi: 10.1016/j.gaitpost.2007.04.002

24. Woollacott M, Shumway-Cook A. Attention and the control of posture and gait: a review of an emerging area of research. Gait Posture (2002) 16:1-14. doi: 10.1016/S0966-6362(01)00156-4

25. Carnahan H, Vandervoort AA, Swanson LR. The influence of summary knowledge of results and aging on motor learning. Res Q Exerc Sport (1996) 67:280-7. doi: 10.1080/02701367.1996.10607955

26. Rouhana J, Ferry F, Toussaint L, Boulinguez P. Knowledge of results and explicit instruction: efficiency of learning the crawl stroke in swimming. Percept Mot Skills (2002) 95:895-6. doi: 10.2466/pms.2002.95.3.895

27. Chiviacowsky S, Wulf G. Feedback after good trials enhances learning. Res $Q$ Exerc Sport (2007) 78:40-7. doi: 10.1080/02701367.2007.10599402

28. Chiviacowsky S, Wulf G, Wally R, Borges T. Knowledge of results after good trials enhances learning in older adults. Res Q Exerc Sport (2009) 80:663-8. doi: 10.1080/02701367.2009.10599606

29. Galea JM, Mallia E, Rothwell J, Diedrichsen J. The dissociable effects of punishment and reward on motor learning. Nature Neurosci. (2015) 18:597602. doi: $10.1038 / \mathrm{nn} .3956$

30. Beck TW. The importance of a priori sample size estimation in strength and conditioning research. J Strength Cond Res. (2013) 27:2323-37. doi: 10.1519/JSC.0b013e318278eea0 
31. Scaglioni-Solano P, Aragon-Vargas LF. Validity and reliability of the Nintendo Wii Balance Board to assess standing balance and sensory integration in highly functional older adults. Int J Rehabil Res. (2014) 37:138-43. doi: 10.1097/MRR.0000000000000046

32. Jorgensen MG, Laessoe U, Hendriksen C, Nielsen OB, Aagaard P. Intrarater reproducibility and validity of Nintendo Wii balance testing in community-dwelling older adults. J Aging Phys Activity (2014) 22:269-75. doi: 10.1123/japa.2012-0310

33. Yasuda K, Kaibuki N, Harashima H, Iwata $H$. The effect of a haptic biofeedback system on postural control in patients with stroke: an experimental pilot study. Somatosens Mot Res. (2017) 34:65-71. doi: 10.1080/08990220.2017.1292236

34. Piirtola M, Era P. Force platform measurements as predictors of falls among older people-a review. Gerontology (2006) 52:1-16. doi: 10.1159/0000 89820

35. Salmoni AW, Schmidt RA, Walter CB. Knowledge of results and motor learning: a review and critical reappraisal. Psychol Bull. (1984) 95:355-86. doi: 10.1037/0033-2909.95.3.355

36. Schmidt RA. Control processes in motor skills. Exerc Sport Sci Rev. (1976) 4:229-61. doi: 10.1249/00003677-197600040-00009

37. MacKenzie CL, Marteniuk RG. Motor skill: feedback, knowledge, and structural issues. Can J Psychol. (1985) 39:313-37. doi: 10.1037/h0080064

38. da Silva RA, Bilodeau M, Parreira RB, Teixeira DC, Amorim CF. Age-related differences in time-limit performance and force platform-based balance measures during one-leg stance. J Electromyogr Kinesiol. (2013) 23:634-9. doi: 10.1016/j.jelekin.2013.01.008

39. Michikawa T, Nishiwaki Y, Takebayashi T, Toyama Y. One-leg standing test for elderly populations. J Orthop Sci. (2009) 14:675-85. doi: 10.1007/s00776-009-1371-6

40. Roemer K, Raisbeck L. Temporal dependency of sway during single leg stance changes with age. Clin Biomech. (2015) 30:66-70. doi: 10.1016/j.clinbiomech.2014.10.010

41. O'Shea S, Morris ME, Iansek R. Dual task interference during gait in people with Parkinson disease: effects of motor versus cognitive secondary tasks. Phys Ther. (2002) 82:888-97. doi: 10.1093/ptj/82.9.888
42. Manor B, Zhou J, Jor'dan A, Zhang J, Fang J, Pascual-Leone A. Reduction of dual-task costs by noninvasive modulation of prefrontal activity in healthy elders. J Cogn Neurosci. (2016) 28:275-81. doi: 10.1162/jocna00897

43. Prieto TE, Myklebust JB, Hoffmann RG, Lovett EG, Myklebust BM. Measures of postural steadiness: differences between healthy young and elderly adults. IEEE Trans Biomed Eng. (1996) 43:956-66. doi: 10.1109/10.532130

44. Lafond D, Corriveau H, Hebert R, Prince F. Intrasession reliability of center of pressure measures of postural steadiness in healthy elderly people. Arch Phys Med Rehabil. (2004) 85:896-901. doi: 10.1016/j.apmr.2003.08.089

45. Ellmers TJ, Cocks AJ, Doumas M, Williams AM, Young WR. Gazing into thin air: the dual-task costs of movement planning and execution during adaptive gait. PLoS ONE (2016) 11:e0166063. doi: 10.1371/journal.pone.0166063

46. Cuypers K, Leenus DJ, van den Berg FE, Nitsche MA, Thijs H, Wenderoth $\mathrm{N}$, et al. Is motor learning mediated by tDCS intensity? PLoS ONE (2013) 8:e67344. doi: 10.1371/journal.pone.0067344

47. Wulf G, Iwatsuki T, Machin B, Kellogg J, Copeland C, Lewthwaite R. Lassoing skill through learner choice. J Mot Behav. (2017). doi: 10.1080/00222895.2017.1341378. [Epub ahead of print].

48. Dijkstra BW, Horak FB, Kamsma YP, Peterson DS. Older adults can improve compensatory stepping with repeated postural perturbations. Front Aging Neurosci. (2015) 7:201. doi: 10.3389/fnagi.2015.00201

49. Peterson DS, Dijkstra BW, Horak FB. Postural motor learning in people with Parkinson's disease. J Neurol. (2016) 263:1518-29. doi: 10.1007/s00415-016-8158-4

Conflict of Interest Statement: The authors declare that the research was conducted in the absence of any commercial or financial relationships that could be construed as a potential conflict of interest.

Copyright (c) 2018 Yasuda, Saichi and Iwata. This is an open-access article distributed under the terms of the Creative Commons Attribution License (CC BY). The use, distribution or reproduction in other forums is permitted, provided the original author(s) and the copyright owner are credited and that the original publication in this journal is cited, in accordance with accepted academic practice. No use, distribution or reproduction is permitted which does not comply with these terms. 